\title{
High-End Silicon PDICs
}

\section{H. Zimmermann}

Institute of Electrical Measurements and Circuit Design, Vienna University of Technology, Vienna, Austria

\begin{abstract}
An overview on integrated silicon photodiodes and photodiode integrated circuits (PDICs) or optoelectronic integrated circuits (OEICs) for optical storage systems (OSS) and fiber receivers is given. It is demonstrated, that by using low-cost silicon technologies high-performance OEICs being true competitors for some III/V-semiconductor OEICs can be realized. OSS-OEICs with bandwidths of up to $380 \mathrm{MHz}$ and fiber receivers with maximum data rates of up to $11 \mathrm{Gbps}$ are described. Low-cost data comm receivers for plastic optical fibers (POF) as well as new circuit concepts for OEICs and highly parallel optical receivers are described also in the following.
\end{abstract}

\section{Introduction}

There is a wide field of applicatons for Opto ASICs. Inexpensive optical receivers with top performance are in great demand. III-V semiconductors normally achieve the best peformance, while Si technologies offer low production costs. For long distance applications, with data rates of more than $40 \mathrm{Gbps}$, it is useful to accept high costs of III-V semiconductors to get the best performance. On the other hand these high costs make them unattractive for the mass markets of short distance applications where lower data rates are adequate, e.g. local-area networks, fiber to/in the home, optical interconnects on and between boards. Nevertheless it is necessary to use advanced circuit design to reach competitive results with Si technologies. Si photodetectors can detect visible and near-infrared light which is used for short distance communications, as well as for plastic-optical-fiber (POF) receivers and in optical pickup units for instance in DVD systems.

Optoelectronic integrated circuits (OEICs) are used for very compact designs and low-cost applications. They com-

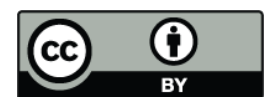

Correspondence to: H. Zimmermann (horst.zimmermann@tuwien.ac.at)

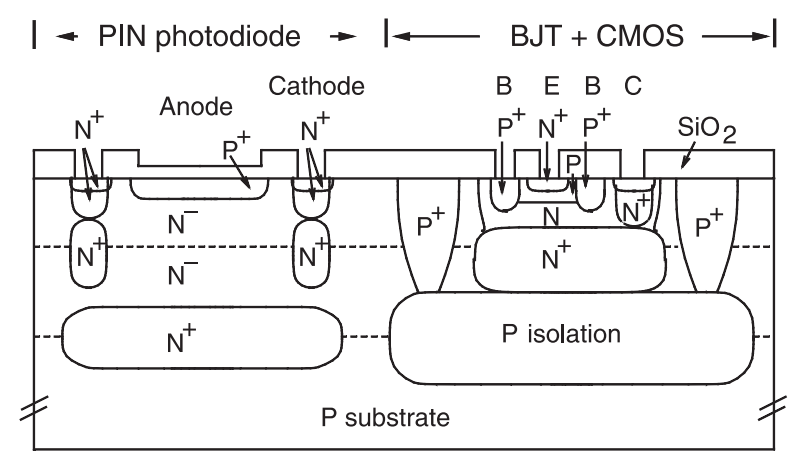

Fig. 1. PIN photodiode integration in standard buried collector (SBC) bipolar technology (Yamamoto, 1995).

bine the photodetector and the receiver circuit on a single chip and therefore mounting and handling are much cheaper and the area consumption and weight is far below a multichip solution. In the next years there will be a demand for high volumes of low-cost highly reliable OEICs for optical buses in cars and in optical storage systems, e.g. CDROM, DVD, Digital Video Recording and Enhanced Video Disk (EVD). For high performance OEICs, pin photodiodes are integrated in Si processes.

\section{Large-diameter integrated-photodiode OEIC}

A vertical PIN photodiode was integrated in a $0.6 \mu \mathrm{m} \mathrm{BiC-}$ MOS process. It is similar in design to the diode presented in (Yamamoto, 1995) which cross section is depicted in Fig. 1. Deep $\mathrm{N}$ plugs after low-doped $\mathrm{N}$-epitaxial steps form the connection from the Si surface to the N+ buried-layer cathode. The electrical isolation between the npn transistors and the $\mathrm{N}$-wells is formed by a P-isolation layer, which separates them. (Yamamoto, 1995) presented a bandwidth of $300 \mathrm{MHz}$ at $780 \mathrm{~nm}$ light with a reverse bias voltage of $3 \mathrm{~V}$. However, the reverse bias voltage can be larger than the chip supply

Published by Copernicus Publications on behalf of the URSI Landesausschuss in der Bundesrepublik Deutschland e.V. 


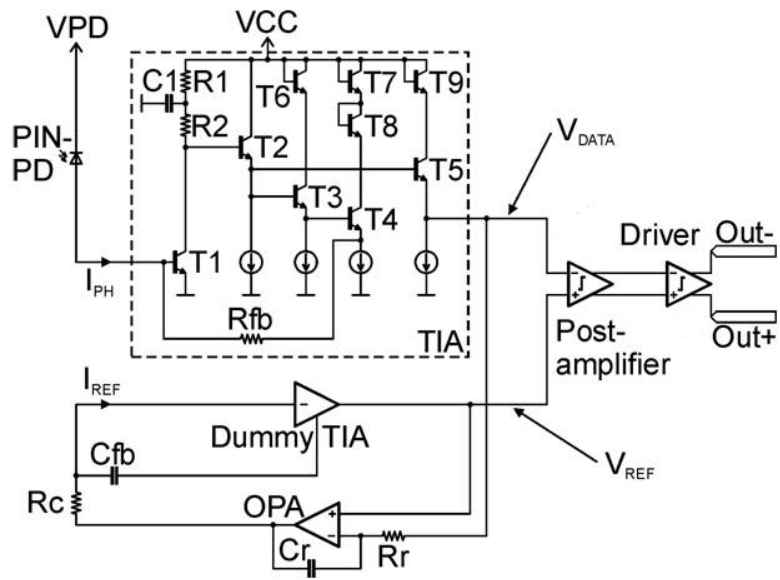

Fig. 2. Schematic of a $2.5 \mathrm{Gbps}$ silicon receiver OEIC with largediameter photodiode (Swoboda, 2004).

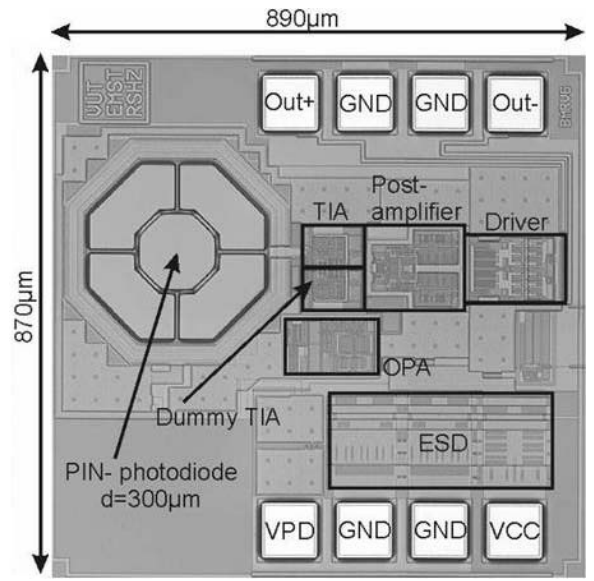

Fig. 3. Microphotograph of a $2.5 \mathrm{Gbps}$ silicon receiver OEIC with large-diameter photodiode (Swoboda, 2004).

voltage of $5 \mathrm{~V}$ due to the fact that the $\mathrm{N}$ cathode is realized in a P-type substrate. This is important to speed up the carrier drift and to increase the bandwidth.

Run with a bias voltage of $12 \mathrm{~V}$, a bandwith of more than $1.35 \mathrm{GHz}$ was reached with a $660 \mathrm{~nm}$ laser diode (Swoboda, 2004). The simplified schematic of this $2.5 \mathrm{Gbps}$ Si receiver OEIC with large-diameter photodiode is depicted in Fig. 2. A resopsivity of $0.36 \mathrm{~A} / \mathrm{W}$ was achieved due to the thick intrinsic layer. In Fig. 3, a microphotograph of the OEIC receiver is depicted. The large photodiode with a diameter of $300 \mu \mathrm{m}$ can be clearly seen. The chip dimensions are $890 \mu \mathrm{m}$ times $870 \mu \mathrm{m}$. The Si OEIC achieved a sensitivity of $-17.2 \mathrm{dBm}$ compared to $-15.7 \mathrm{dBm}$ of a GaAs OEIC (Lang, 2001), whereby the die area of the Si OEIC was one fifth of that of the GaAs OEIC and the power consumption was one third.

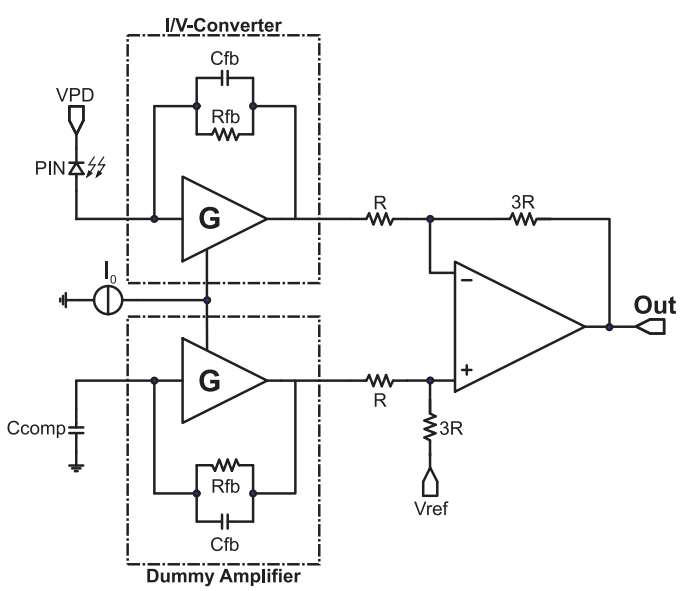

Fig. 4. Schematic of a $380 \mathrm{MHz}$ two-stage OEIC for the use in DVD (Leeb, 2004).

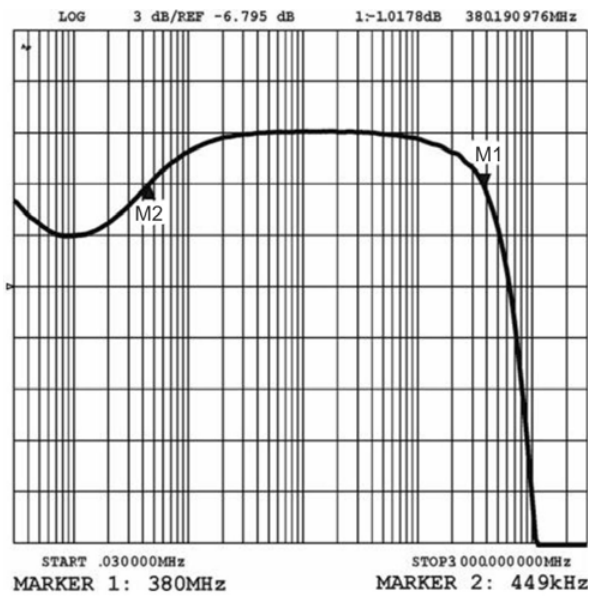

Fig. 5. Frequency response of a $380 \mathrm{MHz}$ two-stage OEIC for the use in DVD (Leeb, 2004).

\section{Two-stage OEIC for the use in DVD}

In (Leeb, 2004) an amplifier architecture that uses a differential transimpedance amplifier in the first stage and an operational amplifier in a second stage is presented.

The used amplifier structure is shown in Fig. 4. The main $\mathrm{I} / \mathrm{V}$ converter and the dummy I/V converter are connected by a current source $\left(\mathrm{I}_{0}\right)$ and therefore both of them contribute gain to the input stage. The second stage is designed as subtractor amplifier and subtracts and amplifies the differential output voltage of the first-stage amplifiers. Furthermore a reference voltage $\left(\mathrm{V}_{\text {ref }}\right)$ is added to reach a single-ended output with low offset voltage compared to $\left(\mathrm{V}_{\text {ref }}\right)$. This architecture together with the capacitance $\left(\mathrm{C}_{\mathrm{COMP}}\right)$ enables a doubled transimpedance with the same value of the feedback resistor $\left(\mathbf{R}_{f b}\right)$ compared to other concepts. This leads to a doubled differential transimpedance compared to the ar- 


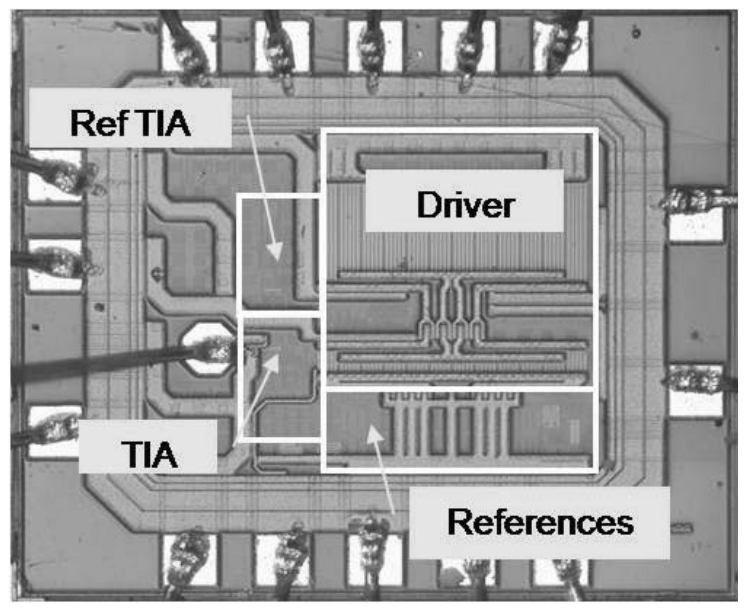

Fig. 6. Schematic of a $130 \mathrm{MHz}$-bandwidth, $120 \mathrm{~dB}$-dynamic-range TIA in $0.35 \mu \mathrm{m} \mathrm{SiGe} \mathrm{BiCMOS} \mathrm{(Micusik,} \mathrm{2007).}$

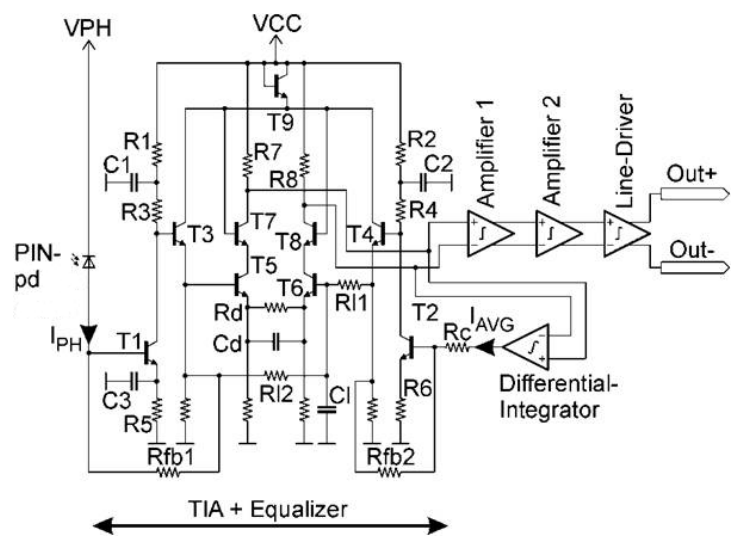

Fig. 7. Micorphotograph of a $130 \mathrm{MHz}-$ bandwidth, $120 \mathrm{~dB}$ dynamic-range TIA in $0.35 \mu \mathrm{m} \mathrm{SiGe} \mathrm{BiCMOS} \mathrm{(Micusik,} \mathrm{2007).}$

chitecture used in (Kieschnick, 1999, Zimmermann, 2000, and Kieschnick, 2003) for the same $\left(\mathbf{R}_{f b}\right)$ value. The bandwidth was increased by a factor of 6.8 compared to a twostage amplifier presented in (Takimoto, 1998) and by a factor of 2.6 compared to (Kieschnick, 1999). In addition, the photo-sensitivity was increased by a factor of about $10 \mathrm{com}-$ pared to (Kieschnick, 1999, Zimmermann, 2000). In total, the transimpedance-bandwidth product was increased by a factor of 26 compared to (Kieschnick, 1999).

The analog modulation port of the network analyzer was used to modulate the laser source for the measurement of the frequnency response. In Fig. 5 the measured frequency response is depicted. It can be seen that the upper $-3 \mathrm{~dB}$ cut-off frequency of the two-stage transimpedance amplifier is $380 \mathrm{MHz}$. The lower cut-off frequency is $450 \mathrm{kHz}$, for frequencies below that lower cut-off frequency the gain drops by $6 \mathrm{~dB}$.

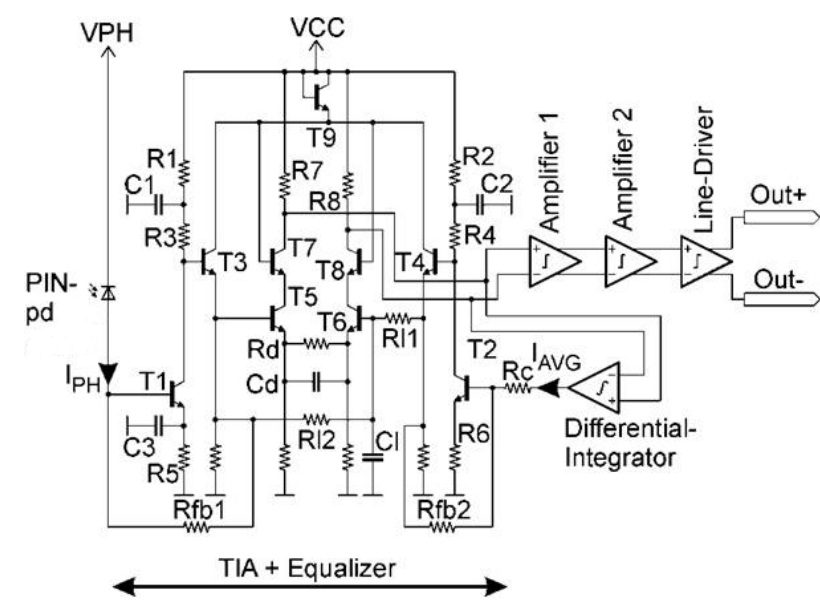

Fig. 8. Simplified schematic of a $11 \mathrm{Gbps}$ optical receiver (Swoboda, 2006).

\section{$4130 \mathrm{MHz}-b a n d w i d t h, 120 \mathrm{~dB}-$ dynamic-range tran- simpedance amplifier (TIA) in $0.35 \mu \mathrm{m}$ SiGe BiC- MOS}

The basic circuit topology of the $130 \mathrm{MHz}-$ bandwidth, $120 \mathrm{~dB}$-dynamic-range TIA in $0.35 \mu \mathrm{m} \mathrm{SiGe} \mathrm{BiCMOS} \mathrm{(Mi-}$ cusik, 2007) with implemented compression is shown in Fig. 6.

The circuit consists of three transistor stages: the first in common-collector configuration (Q0) followed by a common-emitter voltage amplifier (Q1) and as third stage a common-collector voltage buffer $(\mathrm{Q} 2)$. The feedback resistor $\left(\mathrm{R}_{f}=22 \mathrm{k} \Omega\right)$ is connected from the output of the third stage to the input of the first transistor stage. For minimum electronic noise the input common-collector stage (Q0) can be biased without any influence of the following stages. There are two types of operation. In the linear region the transistors Q5 and Q6 are off and therefore do not affect the circuit for small input currents. In the compression region the transistor Q6 is on and provides current to the input and therefore a parallel path to $\left(\mathrm{R}_{f}\right)$ which leads to a lower overall transimpedance of the TIA. Stability over the full input current range is provided by the compensation circuit consisting of Q3, Q4 and Q5. The open-loop gain of the circuit is lowered by the compensation circuit dynamically and therefore the phase margin of the loop gain is kept large enough. In the linear region the TIA itself is the limiting element regarding to the bandwidth (BW). In the compensation region the BW of the TIA increases and the limiting elements are the following stages, e.g. the differential $50 \Omega$ driver. The full width at half maximum (FWHM) of the input current pulses used for characterization was $10 \mathrm{~ns}$ with a rise and fall time of $1.5 \mathrm{~ns}$. The circuit proposed in (Micusik, 2007) exhibits the highest dynamic range $(120 \mathrm{~dB})$ for the input current compared to the literature. The power consumption of 


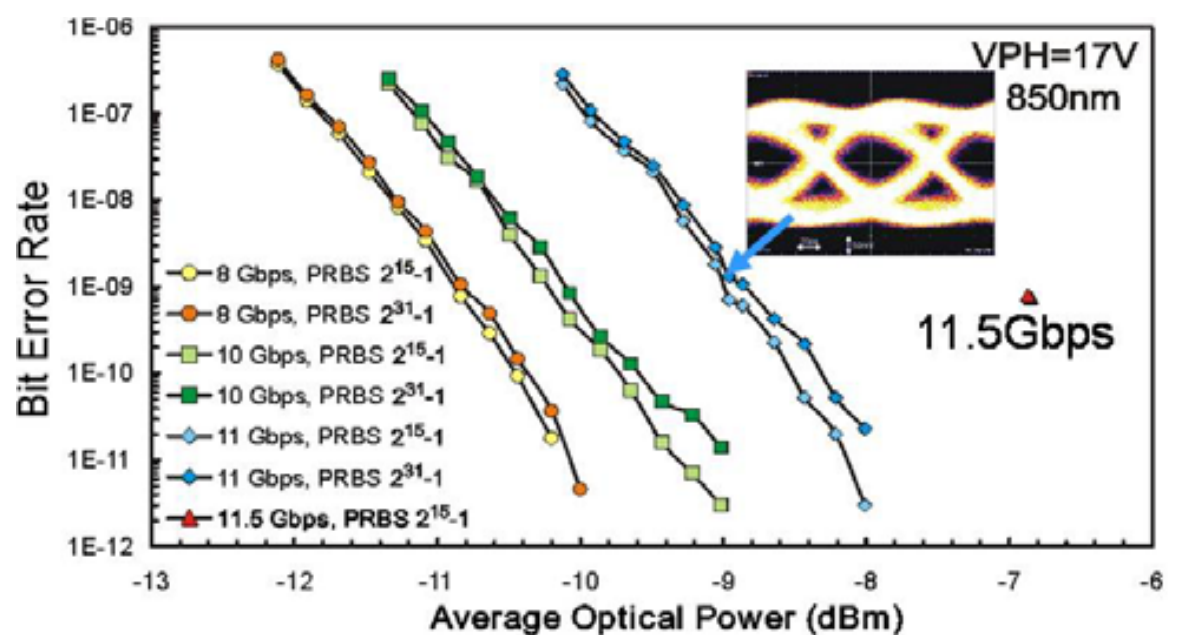

Fig. 9. Bit error rate vs. average optical power of a $11 \mathrm{Gbps}$ optical receiver (Swoboda, 2006).

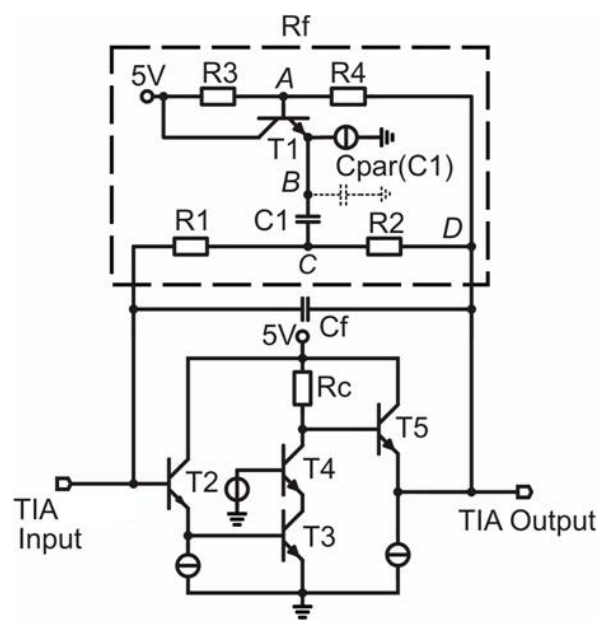

Fig. 10. Schematic of a 1-stage transimpedance amplifier with CCVD (Seidl, 2004).

the TIA alone is $2 \mathrm{~mA}$ from a $5 \mathrm{~V}$ power supply. The complete receiver circuit including a $50 \Omega, 1.7 \mathrm{~V}$ peak-to-peak differential output driver consumes an area of $1.24 \mathrm{~mm}^{2}$ in $0.35 \mu \mathrm{m} \mathrm{SiGe} \mathrm{BiCMOS} \mathrm{technology} \mathrm{and} \mathrm{the} \mathrm{complete} \mathrm{power}$ consumption is $390 \mathrm{~mW}$.

A microphotograph of the $130 \mathrm{MHz}$-bandwidth, $120 \mathrm{~dB}$ dynamic-range TIA in $0.35 \mu \mathrm{m}$ SiGe BiCMOS is depicted in Fig. 7.

\section{Gbps optical receiver}

(Swoboda, 2006) presented a monolithically integrated silicon optical receiver for $850 \mathrm{~nm}$ light. The used photodi-

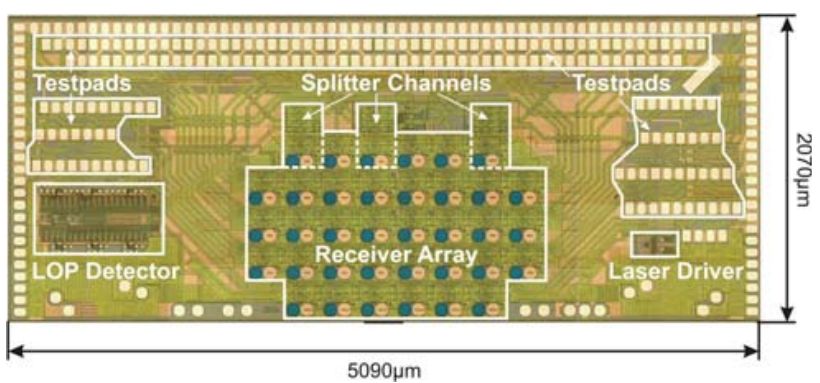

Fig. 11. Highly parallel optical receiver (Swoboda et al, 2006).

ode has a diameter of $50 \mu \mathrm{m}$ and therefore matches exactly a $50 \mu$ m multimode fiber.

The schematic of the input circuit including a simplified schematic of the following stages is depicted in Fig. 8. A TIA consisting of T1, T3, R1, R3, R5, C1, C3, and Rfb1 is used to convert the photo current into an output voltage. The transimpedance is $500 \Omega$. The output voltage of the TIA subtracted by a reference voltage is amplified frequency dependent. The gain has the inverse characteristic to the photodiode frequency response. This is realized by a cascode difference amplifier consisting of T5, T6, T7, T8, R7 and R8 including frequency-dependent coupling elements. $\mathrm{Rd}$ and Cd compensate the photodiode drift time and the small diffusion part is compensated with $\mathrm{C} 1, \mathrm{R} 11$ and R12. A nearly flat frequency response with a linear phase is reached with this equalizer. TIA and equalizer are followed by three limiting amplifiers to improve the gain and drive a differential $100 \Omega$ load. The average photo current $\left(\mathrm{I}_{\mathrm{AVG}}\right)$ generated by a differential integrator, a controlling circuit and a dummy TIA is provided to the dummy TIA via Rc. Therefore symmetrical clipping of the " 0 " $s$ and " 1 " $s$ is provided in case of 


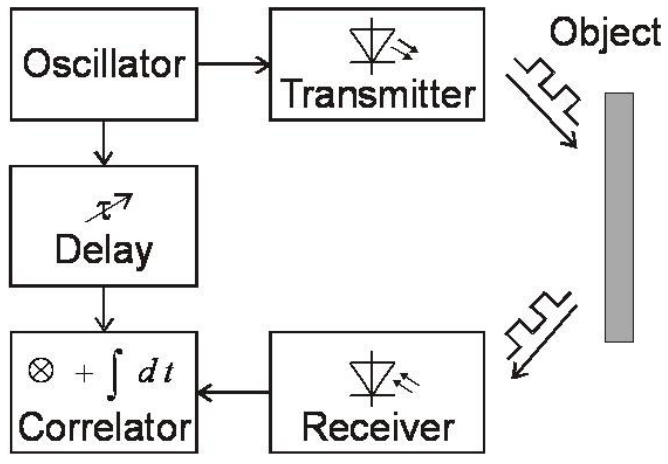

Fig. 12. Block diagram of optical distance measurement/3-D camera with continuous wave system.

optical overdrive. The background light is suppressed by a lower cut-off frequency of $30 \mathrm{kHz}$ introduced by the controlling circuit.

The bit-error rate (BER) was measured with a bit error analyzer for different data rates, optical power levels, and two different lengths of the pseudo random bit sequence (PRBS). The summarized results are depicted in Fig. 9. An eye diagram at $11 \mathrm{Gbps}$ and a PRBS of $2^{31}-1$ at minimum optical power of $-8.9 \mathrm{dBm}$ for a BER of $10^{-9}$ is shown in the same figure.

\section{TIA with CCVD feedback}

In conventional TIA topologies in integrated circuits the high-ohmic feedback resistor can be the bandwidth limiting element due to parasitic capacitances to the substrate. To avoid this, a new concept was presented in (Seidl, 2004). It introduces a capacitive-coupled voltage divider (CCVD) instead of an ohmic resistor in the feedback path of the TIA, like depicted in Fig. 10. With this new topology the bandwidth was raised from $67 \mathrm{MHz}$ to $387 \mathrm{MHz}$. Both topologies were realized for better comparison.

The CCVD TIA offers the opportunity to realize higher bandwidth-transimpedance products in one amplifier stage than others known from the literature did in 2- or 3-stage TIAs (Hehemann, 2002). This results in less chip area, less power consumption and less offset of TIAs.

\section{Highly parallel optical receiver OEIC}

In (Swoboda et al, 2006) a highly parallel optical receiver OEIC in a $0.6 \mu \mathrm{m} \mathrm{Si} \mathrm{BiCMOS} \mathrm{technology} \mathrm{was} \mathrm{presented.} \mathrm{It}$ contains 36 channels with integrated PIN photodiode and a data rate of $3 \mathrm{Gbps}$ each. This very compact design with a pitch of $250 \mu \mathrm{m}$ requires a special layout of the receiver to minimize crosstalk. The measured sensitivity at $3 \mathrm{Gbps}$ is $-17 \mathrm{dBm}$ at $850 \mathrm{~nm}$ in each channel. This means that the

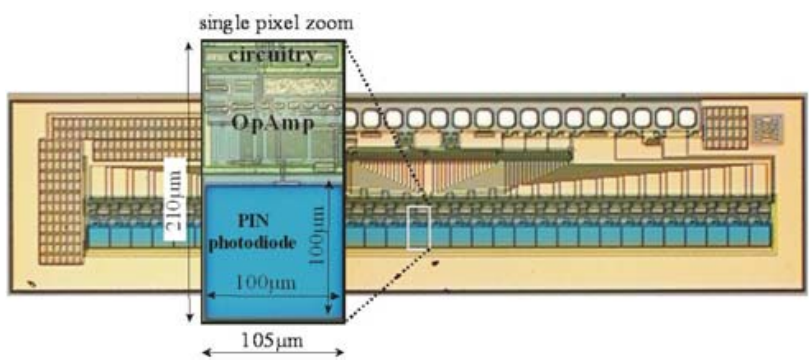

Fig. 13. Microphotograph of a line sensor for optical distance measurement with a continuous-wave system.

presented receiver OEIC offers an over-all data rate of more than $100 \mathrm{Gbps}$ combined with a matching laser array on the sender side. Figure 11 shows a microphotograph of a test chip for the receiver chip.

The power consumption of the OEIC is $809 \mathrm{~mW}$ including that of a laser driver for a return channel and a loss-ofpower detector. The crosstalk between the channels is below $-40 \mathrm{~dB}$.

\section{Optical distance measurement}

Sensors for capturing 3-D information of a scene in real time i.e. in a few milliseconds are still not available on the lowcost market on a grand scale. Mass products, for example neighbour-lane supervision or passenger recognition airbag control in a modern car, can use them. The interesting distance range for these sensors is form $\mathrm{cm}$ up to some meters. It is important for robust low-cost sensors to avoid moving mechanical parts. The integrated optical distance measurement sensors presented here work on the principle of timeof-flight. It is based on the measurement of runtime of modulated light from a laser source to the surface of a diffuse reflecting object and back to the receiver (see Fig. 12). The main difficulties of this measurement principle are the very weak amplitude of the received signal, especially compared to the high backround light, as well as the short time intervals to be measured. The optical power of the laser is limited for eye-safety reasons to the $\mathrm{mW}$ range. This leads to received signals per pixel in the $\mathrm{nW}$ range only. On the other hand, the time interval to be measured is in the ps range, e.g. the runtime of light to an object $10 \mathrm{~m}$ away from the sensor $\left(\mathrm{c}_{0}=2.998 \times 10^{8} \mathrm{~m} / \mathrm{s}\right)$ is $66.7 \mathrm{~ns}$ which leads to time intervals of $66.7 \mathrm{ps}$ for a resolution of $10 \mathrm{~mm}$. To realize the measurement of these short time intervals the transmitting laser diode is $10 \mathrm{MHz}$ square-wave modulated. The distance is determined out of the phase shift between sent and received signal. The correlation of the received light and the transmitted signal therefore provides the distance information. (Nemecek, 2006) describes this method in more detail. 
Figure 13 shows a microphotograph of a line sensor which consists of 32 single pixels. Results of one pixel were published in (Nemecek et al, 2006). For a measurment time of $5 \mathrm{~ms}$ at an object distance of $3.2 \mathrm{~m}$, a standard deviation of $3 \mathrm{~cm}$ was achieved with an optical power of $2 \mathrm{~mW}$ at $650 \mathrm{~nm}$. The area of each pixel was $210 \times 105 \mu \mathrm{m}^{2}$, whereby the photodiode occupied an area of $100 \times 100 \mu \mathrm{m}^{2}$ in each pixel.

\section{Conclusions}

OEICs for different application areas were presented. The presented work of the circuit design group of the Institute of Electrical Measurements and Circuit Design of Vienna University of Technology is on top of the state of the art. E.g. the achieved data rate of $11 \mathrm{Gbps}$ with a Si OEIC is the highest possible with a thick vertical Si photodiode having a quantum efficiency close to $100 \%$. Another quantum leap was reached with the capacitive-coupled voltage driver (CCVD) feedback. It enables top performances of single-stage TIAs, without the stability problems of multi-stage TIAs. Another outstanding optical receiver is the highly parallel receiver OEIC which enables a data rate of more than $100 \mathrm{Gbps}$ with a low-cost Si BiCMOS technology. A 32 pixel line sensor for distance measurement shows the potential of Si OEICs to realize a 3-D camera on a chip. All in all it could be demonstrated that high-performance low-cost Opto ASICs for a wide variety of high-volume applications are possible with BiCMOS OEICs.

Acknowledgements. The author thanks the members of the circuit design group at EMST of TU Vienna and R. Swoboda, M. Förtsch and J. Leeb from A3PICs for careful work. Financial funding in the EU project INSPIRED, the FIT-IT project HYBOR as well as the A3-projects 3-D-Sensors and SENSAFE is acknowledged. Last but not least, many thanks for the great help with preparing this manuscript go to K. Schneider-Hornstein.

\section{References}

Yamamoto, M., Kubo, M., and Nakao, K., et al.: Si-OEIC with a built-in pin-photodiode, IEEE T. Electron. Dev., 42, 58-63, 1995.

Swoboda, R. and Zimmermann H.: 2.5Gbit/s silicon receiver OEIC with large diameter photodiode, Electron. Lett., 40, 505-507, 2004

Lang, M., Bronner, W., Benz, W., et al.: Complete monolithic integrated $2.5 \mathrm{Gbit} / \mathrm{s}$ optoelectronic receiver with large area MSM photodiode for $850 \mathrm{~nm}$ wavelength, Electron. Lett., 37, 20, 1247 1249,2001

Leeb, J., Schneider, K., and Zimmermann, H.: A 380MHz twostage OEIC for the use in DVD pickup units, Proceedings of IEEE International Symposium on Consumer Electronics, 381384, 2004.

Kieschnick, K., Heide, T., Ghazi, A., and Zimmermann, H.: Advanced CMOS and BICMOS Photonic Receiver ICs, Proceed- ings of European Solid-State Circuits Conference (ESSCIRC), 398-401, 1999.

Zimmermann, H. and Kieschnick, K.: Low-Offset BiCMOS OEIC for Optical Storage Systems, Electron. Lett., 36, 1223-1224, 2000.

Kieschnick, K. and Zimmermann, H.: High-Sensitivity BiCMOS OEIC for Optical Storage Systems, IEEE Journal of Solid-State Circuits, 38, 579-584, 2003.

Takimoto, T., Fukunaga, N., Kubo, M., and Okabayashi, N.: High Speed SI-OEIC (OPIC) for optical pickup, IEEE Transactions on Consumer Electronics, 44, 137-142, 1998.

Micusik, D. and Zimmermann, H.: Transimpedance amplifier with $120 \mathrm{~dB}$ dynamic range, Electron. Lett., 43, 3, 159-160, 2007.

Swoboda, R. and Zimmermann, H.: $11 \mathrm{~Gb} / \mathrm{s}$ Monolithically Integrated Silicon Optical Receiver for 850nm Wavelength, IEEE International Solid-State Circuit Conference, Digest of Technical Papers, ISSCC, 49, 0193-6530, 240-241, 2006.

Seidl, C., Knorr, J., and Zimmermann, H.: Single-stage $378 \mathrm{MHz}$ $178 \mathrm{k}$-transimpedance amplifier with capacitive coupled voltage divider, IEEE ISSCC, 470-471 and 540, 2004.

Hehemann, I., Brockherde, W., Bussmann A., Hoffmann, H., Kemma, A., Kokzinski, R., Richter, H., and Hosticka, B. J.: A new detector architecture for optical pickup units in DVD systems, IEEE Int. Symp. on Circuits and Systems (ISCAS), 4, 4144, 2002.

Swoboda, R., Förtsch, M., and Zimmermann, H.: 3 Gbps-perChannel Highly-Parallel Silicon Receiver OEIC, Proc. 33rd European Conference and Exhibition on Optical Communication (ECOC), 2, 255-256, 2007.

Nemecek, A., Oberhauser, K., and Zimmermann, H.: Distance Measurement Sensor With PIN-Photodiode and Bridge Circuit, IEEE Sensors Journal, 6, 2, 391-397, 2006

Nemecek, A., Oberhauser, K., Zach, G., and Zimmermann, H.: Distance Measurement Line Sensor with PIN Photodiodes, IEEE Sensors Conference, 275-278, 2006. 